\title{
THE AUSTRALIAN MUSEUM, SYDNEY
}

MEMOIR X.

\section{CATALOGUE OF THE CERAMBYCIDAE (COLEOPTERA) OF AUSTRALIA}

By

KEITH C. McKEOWN, F.R.Z.S.,

Assistant Entomologist, The Australian Museum.

PUBLISHED BY ORDER OF THE TRUSTEES

A. B. Walkom, D.Sc., Director.

Sydney, May 2, 1947 


\section{PREFACE.}

The accompanying Catalogue of the Cerambycidae is the first, dealing solely with Australian genera and species, to be published since that of Pascoe in 1867. Masters' Catalogue of the Described Coleoptera of Australia, 1885-1887, included the Cerambycidae, and was based on the work of Gemminger and Harold.

A new catalogue has been badly needed owing to the large number of new species described in recent years, and the changes in the already complicated synonymy. The Junk catalogue, covering the Coleoptera of the world, is defective in many respects, as well as being too unwieldy, and too costly for the average Australian worker. Many of the references in the Junk catalogue are inaccurate, synonymy misleading, and the genera under which the species were originally described omitted, and type localities are not quoted.

In this catalogue every care has been taken to ensure accuracy, and the fact that it has been used, in slip form, over a number, of years as a working list has been invaluable in detecting and correcting errors. Several features not previously to be found in any catalogue have been included. The genotype has been determined in each instance, recorded localities have been included, as well as the range of the genera, and the genera under which the species were originally described. Other references not hitherto included are those referring to geographical distribution of species, and on economic questions, as also are the recorded food-plants. In view of the economic importance of the Cerambycidae in forestry, the value of the latter cannot be too strongly stressed.

Species doubtfully of Australian origin, or of which the validity is questionable, have been retained in the list, and are enclosed in square brackets, for the convenience of workers.

I am indebted to the late Dr. Walther Horn for considerable advice and assistance regarding the location of types-another feature not hitherto included in a catalogue. 